\title{
El desafío del gigante chino a Estados Unidos en la posguerra fría: ¿mito o realidad?*
}

\section{Alejandra RipolI** Rafat Ghotme}

\author{
No importa que el gato sea blanco o sea negro. \\ Mientras cace ratones, es un buen gato. \\ Deng Xiao Ping
}

\section{RESUMEN}

Tras veinticinco años de haber finalizado la Guerra Fría, el balance del ascenso de China en el sistema internacional se puede analizar desde elementos materiales e ideacionales de poder. El objetivo de este artículo es identificar si la importancia de China internacionalmente está sobrevalorada, o si realmente puede lle- gar a desafiar la hegemonía estadounidense. La mayoría de la literatura sobre el potencial chino ha dejado de lado su política interna, factor determinante en el sistema internacional, según el realismo. Desde el método inductivo se intentará establecer la relación causal entre las políticas doméstica e internacional, para sustentar la necesidad china de resolver los diferendos limítrofes con sus

* Este artículo pertenece al grupo de investigación Estudios Internacionales y Políticos en la línea Relaciones Internacionales, reconocido por Colciencias, Bogotá (Colombia). Proyecto Dejando a Westfalia. eEs311.

** Magíster en Relaciones Internacionales. Profesora asociada e investigadora de la Facultad de Relaciones Internacionales, Estrategia y Seguridad, Universidad Militar Nueva Granada, Bogotá (Colombia) [alejandra.ripoll@ unimilitar.edu.co].

*** PhD en Historia Política Comparada. Profesor asociado e investigador de la Facultad de Relaciones Internacionales, Estrategia y Seguridad, Universidad Militar Nueva Granada; Investigador del Centro Colombiano de Estudios Árabes, Bogotá (Colombia) [Rafat.ghotme@utadeo.edu.co].

Recibido: 1 de abril de 2016/ Modificado: 6 de abril de 2016/ Aceptado: 7 de abril de 2016

Para citar este artículo

Ripoll, A. y Ghotme, R. (2017). El desafío del gigante Chino a Estados Unidos en la posguerra fría: ¿̨mito o realidad? OASIS, 25, 115-127.

DOI: https://doi.org/10.18601/16577558.n25.07 
vecinos, comprometerse por el respeto de los derechos humanos y cubrir las necesidades de una población altamente insatisfecha, debido a la inequidad y la corrupción. Estos factores inciden para que China adopte una política exterior prudente, para equiparar su posición relativa de poder frente a Estados Unidos.

Palabras clave: China, ascenso, poder, política, economía.

\section{The challenge of the chinese giant to the U.S. in the post cold war period: myth or reality?}

\section{ABSTRACT}

After twenty-five years of the end of the Cold War, the impact of the rise of China in the international system can be analyzed from material and ideational elements of power. This article seeks to identify whether China's importance is internationally overrated, or if this country can really challenge U.s. hegemony. Most of the literature on Chinese potential has overlooked its internal policy, which is a determining factor in the international system, according to the realist perspective. Using the inductive method, this paper will attempt to establish the causal relation between domestic and international politics. This will highlight China's need to solve border disputes with its neighbors, make a commitment to respect human rights, and solve the needs of a population that is highly dissatisfied with the present government due to inequality and corruption. These factors challenge China in its quest to achieve a new distribution of power.

Key words: China, ascent, power, politics, economics.

\section{INTRODUCCIÓN}

En los últimos tiempos, uno de los temas de interés para los estudiosos de las relaciones internacionales ha sido el ascenso de China en el sistema internacional. Este país, desde la década de los noventa, buscó centrar y limitar su proceso de modernización en el campo económico, para así satisfacer las necesidades de la población y no acudir a reformas políticas que amenazaran la estabilidad del partido único comunista.

Así mismo, desde esta década buscó reacomodarse en el paso de un sistema bipolar a uno unipolar, en un ambiente dominado por el triunfo del capitalismo y la democracia sobre el comunismo. China comenzó a ser visto como un gigante, llevando a creer que podría convertirse en la competencia directa de Estados Unidos y que podía poner en peligro su hegemonía. Con la expansión de la influencia China en el mundo también se llevaron a cabo esfuerzos para promover su agenda de libre comercio, democracia y derechos humanos, generando un sistema inestable e inseguro ante una nueva distribución del poder.

Desde la política reformista de Deng Xiaoping, sin embargo, se ha venido frenando a los partidarios de una mayor democratización, quienes fueron perseguidos, encarcelados u obligados a salir del país. La línea dura 
conservadora, que quería mantener el statu quo, y los hechos de la Plaza Tiananmen en 1989 permearon las decisiones de los gobernantes chinos frenando mayores reformas económicas.

Como consecuencia de ello, las potencias económicas suspendieron los intercambios comerciales durante unos años. Después de un tiempo la situación fue superada y finalmente, para 1992, Japón -junto con los países de la Unión Europea y Washington- restableció las relaciones comerciales con China por las ventajas económicas que representaba para los inversores extranjeros. Washington, por su parte, también lo hizo por el temor de verse desplazado del gran mercado chino. La reanudación de las relaciones comerciales le inyectó un dinamismo a la economía china que contribuyó decididamente a su crecimiento.

En este artículo se sostendrá que a pesar del acelerado crecimiento económico y de la influencia que China ha logrado en el sistema, esta potencia ha implementado una política prudente y pragmática con el objetivo de salvaguardar sus intereses nacionales y expandir su influencia, sin desafiar claramente la hegemonía estadounidense. El objetivo de este artículo es identificar si China tiene los elementos de poder para retar la hegemonía estadounidense o si, por el contrario, este fenómeno ha sido exagerado por los analistas y estudiosos. Desde el realismo político se analizará cómo la situación interna tiene efectos en el posicionamiento de una potencia en el sistema internacional. Por tanto, desde el método inductivo se estudiarán los factores de política interna para llegar a deducir que los problemas de política doméstica inciden para que puedan cambiar la distribución de poder.

\section{PROSPERIDAD Y EXPANSIÓN CHINA EN LOS NOVENTA}

La década de los noventa se torna favorable para China, porque el ambiente internacional, que se estaba reacomodando por el paso de un sistema bipolar a uno unipolar, determinado por el triunfo del capitalismo y la democracia sobre el comunismo, era propicio para que actuara con menos restricciones, lo que hizo que China fuera vista como un gigante. Algunos autores afirmaron que podría convertirse en la competencia directa de Estados Unidos y que podría poner en peligro su hegemonía. Es por eso que la expansión de la influencia China en el mundo, en lo comercial y lo energético, y en menor medida en lo militar, sea presentada como una estrategia muy incómoda para Estados Unidos y sus aliados tradicionales. Esto supone que podría desplazar a la gran potencia, minando sus esfuerzos para promover su agenda de libre comercio, democracia y derechos humanos y, por tanto, generando un sistema inestable o inseguro ante una nueva distribución del poder (Ellis, 2013, p. 140; 2009).

Esta tesis es válida, pero no se debería exagerar el nivel de amenaza o incertidumbre que se presenta en el corto plazo, porque Estados Unidos, en lugar de rivalizar con China, está intentando sacar provecho de esta situación, teniendo en cuenta que debido a su crecimiento económico y militar tiene mayores presiones en cuanto a medio 
ambiente, crecimiento de la población, freno a la emigración, diferencias en el nivel de vida entre las distintas regiones del país y la administración eficiente de las empresas del Estado. Estas demandas de política interna no son fáciles de sortear, impidiendo que el nivel de alcance chino tenga un carácter mundial.

Además, porque en la misma década varias organizaciones como Human Rights Watch, Amnistía Internacional e incluso el Departamento de Estado de Estados Unidos la cuestionaron por la violación sistemática de los derechos humanos, especialmente por la falta de libertad de expresión, movimiento y religión de la población. A estas acusaciones los chinos respondieron que reconocían que había algunas fallas en el sistema pero que estaban mejorando. Este tipo de reconocimiento también pone sobre la mesa la debilidad china.

En los años siguientes, la situación no cambió y aún se les acusa de violar frecuente y sistemáticamente los derechos humanos de su población. Según el World Report 2013 de Human Rights Watch, en China no se garantizan los derechos y las libertades civiles. Agregan que cualquier manifestación por inconformidad en contra del régimen es rebatida con encarcelamiento y tortura. El autoritarismo político, que tiene que ver con el control político en manos del Partido Comunista Chino, prohíbe la libertad de opinión, acompañado de una constante persecución y restricción política. Uno de los casos documentados es el del abogado defensor de los derechos humanos $\mathrm{Pu}$ Zhiqiang, que fue detenido el 5 de mayo de 2014 y acusado por subversión después de haber asistido a una conmemoración privada de las protestas de Tiananmen, junto a otros casos documentados.

A pesar de las acusaciones de violaciones de derechos humanos y el aumento de la presión socioeconómica, internacionalmente ha ganado reconocimiento universal y se ha constituido como potencia regional y mundial. Esa nueva China busca la compatibilidad entre nacionalismo y modernización, que se convierte en la base de su política exterior, con repercusiones mundiales y regionales (Quansheng, 2001, p. 66). En consecuencia, regionalmente se hará más fuerte, intentando una política de mayor integración económica a través de mecanismos de cooperación. Sin embargo, ese liderazgo se logrará en caso de que Rusia y Estados Unidos asuman perfiles más bajos. Pero si se toma en consideración el comportamiento de Obama de los últimos tiempos, con un marcado acento realista, se complican más las cosas para las aspiraciones de China. En esa medida, no se puede garantizar que su desarrollo siga enmarcado en un ambiente pacífico como ha sido hasta ahora, y que ha caracterizado la sucesión de los dirigentes chinos, porque los nuevos líderes se enfrentan al reto de responder a las necesidades de la nueva China.

El ambiente pacífico en el que se ha desarrollado China puede verse turbado, entre varias causas, debido a la complejidad que rodea sus relaciones con algunos de sus vecinos. Por ejemplo, con India, Japón, Vietnam y Filipinas tiene problemas por reivindicaciones territoriales. A su vez, India, Pakistán y Bangladesh mantienen una alta conflictividad, que afecta el liderazgo que China quiere 
mantener en la región, a pesar de la actitud amable y la buena voluntad política que ha demostrado recientemente Li Keqiang ${ }^{1}$. Las relaciones China-Vietnam también tienen un alto nivel de complejidad debido a la asociación geoestratégica de este último con India para la exploración petrolera.

En fin, este panorama confirma lo que han dicho autores como García (2001) y Velosa (2010) acerca de que en Asia no se ha terminado la Guerra Fría. Por esa razón, China le da más importancia a la seguridad que a la oportunidad económica (Quansheng, 2001, p. 66), no solo por los conflictos mencionados, sino por la capacidad militar que han desarrollado sus vecinos indios, pakistaníes y la propia Federación Rusa, que tienen armamento nuclear y pueden desequilibrar la región. A pesar de este panorama enrarecido, China unida a Japón, a pesar de sus rivalidades, pueden ayudar a estabilizar la zona, no solo con acuerdos de entendimiento y amistad, sino con la recuperación económica de la región con mayor grado de autonomía respecto a los poderes occidentales, lo que bajaría considerablemente la tensión.

En este sentido, Estados Unidos juega un papel importante en estas relaciones porque tiene interés en mantener el balance clásico de poder en Asia-Pacífico (Quansheng, 2001, p. 41). Pero las cosas no se le tornan tan simples porque algunos sectores de Esta- dos Unidos proponen que se trate a China como a la Unión Soviética en la época de la Guerra Fría, tanto por sostener un régimen comunista como por la violación sistemática de los derechos humanos. Sin embargo, la Casa Blanca comprende que "sería mejor la cooperación por el papel que juega China en lo global” (García, 2001, p. 90).

Otro aspecto importante para tener en cuenta es el crecimiento económico acelerado que ha tenido China, el cual ha sido impulsado por las variables del capitalismo y monopolio estatales. Es por eso que se puede decir que el liderazgo chino promueve, desde el discurso y de la práctica, un ambiente internacional pacífico en el que se consolida su crecimiento económico y además puede garantizarse el acceso a los recursos naturales y energéticos. Al mismo tiempo, intenta buscar un comercio organizado, con una nueva agenda en la Organización Mundial del Comercio (oMc) ${ }^{2}$, con políticas discriminatorias industriales $-\mathrm{y}$ el desconocimiento de las patentes- contrarias de las existentes y propagadas por Europa, Japón y Estados Unidos. En ese mismo sentido, también aspira desempeñar un gran rol en otras organizaciones internacionales tratando de instaurar nuevas reglas de juego (Economy, 2010).

A pesar de que desde 2009 Estados Unidos y China mantienen regularmente un diálogo económico y estratégico, y del len-

\footnotetext{
$1 \quad$ Li Keqiang es, desde marzo de 2013, el primer ministro y secretario del Partido en el Consejo de Estado de la República Popular China.

2 En el 2001, después de trece años de negociación, con el objetivo claro de ser reconocida como país desarrollado y pisar fuerte en la arena política mundial, China se convierte en miembro de la omc.
} 
guaje moderado usado por Estados Unidos en la pasada Cumbre del Foro de Cooperación Económica Asia-Pacífico (APEC-2014), cuando solicitó a los chinos que permitieran la libre competencia, la tensión continúa y se agrava más por las acusaciones mutuas de espionaje, situaciones que si bien no favorecen a ninguno de los contrincantes, Estados Unidos promulga y justifica con su discurso de un mundo seguro.

\section{EL PODER INTELIGENTE CHINO}

La ampliación de las relaciones económicas y políticas-militares de China en el mundo, en los últimos años, ha estado destinada a incrementar su cuota de poder, con el fin de lograr un equilibrio y más adelante desplazar la hegemonía estadounidense. Sin embargo, el rasgo característico que ha identificado el comportamiento chino ha estado marcado por el uso del poder suave, tendiente a conseguir la buena voluntad, la comprensión y la aceptación de su influencia política, a fin de crear las herramientas suficientes para proteger sus intereses en su territorio. Los intereses inmediatos que quiere asegurar son el acceso a materias primas, seguridad alimentaria, protección y seguridad de los mercados para los productos chinos. Igualmente, el acceso a los flujos de información y tecnología, lograr y mantener su presencia en instituciones internacionales clave, buscar posicionarse estratégicamente de forma tal que no provoque a Estados Unidos y sus aliados occidentales; de esa forma, China busca evitar que surja una coalición internacional que se oponga a su ascenso mundial. Estos objetivos implican la promoción y la protección de una política tendiente a su reaparición como una importante potencia mundial.

En este punto es importante tener en cuenta que en la década pasada, el entonces presidente Hu Jintao se refirió al rol de los militares como una "nueva misión en el nuevo siglo", a fin de fomentar "un entorno de seguridad propicio para el desarrollo pacífico de China”. Por tal razón es que los medios políticos y académicos chinos están hablando de una nueva "frontera de interés", es decir, que el Ejército de Liberación Popular chino no solo tiene la obligación de defender los intereses chinos en el territorio físico, sino de protegerlos fuera de su país (Ellis, 2013). Esto significa la declaración de una pretensión de alcance mundial, dejando atrás el aislacionismo que ha caracterizado a la China Comunista y retando así los intereses de Estado Unidos gradualmente.

Sin embargo, existen circunstancias que se interponen a un verdadero activismo internacional chino, relacionadas con las amenazas, los conflictos internos y los problemas estructurales que pueden frenar la carrera de China hacia la hegemonía. Dentro del primer grupo se encuentra un problema que no se ha podido resolver en más de 60 años: su dominio en el territorio del Tíbet, y, en esa misma dirección, el conflicto en Xinjiang. En el grupo dos del abanico de amenazas existen varios factores de riesgo como la superpoblación de china, que cuenta aproximadamente con 1300 millones de habitantes (Banco Mundial, 2015), lo cual la convierte en una potencia demográfica pero 
al mismo tiempo compromete la propia seguridad alimentaria del país y el futuro laboral de millones de personas.

Si bien China ha crecido en los últimos cinco ańos a una tasa promedio del $8 \%$ anual $(9,2 \%$ en $2011 ; 7,8 \%$ en $2012 ; 7,7 \%$ en 2014 y $6,9 \%$ en 2015 ), este crecimiento no es suficiente para satisfacer las necesidades de la enorme tasa de población. Esta es la razón por la que el Gobierno chino aprobó años atrás la política de "hijo único" con el deseo de poder poner coto al crecimiento desenfrenado de la población (Pérez, 2012; World Bank, 2015).

Otra amenaza que no solo es de carácter interno, sino externo, es el deterioro medioambiental causado por el desarrollo industrial y el crecimiento exponencial que ha tenido, que produce altos índices de contaminación y estos a su vez producen todo tipo de enfermedades, con consecuencias nefastas para las poblaciones más vulnerables.

En ese mismo orden de ideas, y no menos importante, la corrupción por parte de miembros del Gobierno y empresarios le resta confianza a la población en sus gobernantes y, por último, debido a la corrupción y a una pésima distribución de los recursos se encuentra la inequidad. El problema de inequidad, a su vez, engendra otro: el resentimiento en la población debido a que existe una brecha muy grande entre los que tienen y los que no. Las zonas costeras tienen mejores niveles de vida, crecimiento en los servicios y mayor industrialización, mientras que otras zonas como los desiertos están lejos de mejorar el nivel de vida. Esas desigualdades provocan y terminan en revueltas como las de 2008 y 2009 en Tibet y Xinjiang.
Estas semillas de subversión han germinado, complicando la gobernanza, y han mermado el control que históricamente ha mantenido el partido de Gobierno. A pesar de ello, el Gobierno ha reaccionado rápidamente y ha protegido el statu quo interno, sin mucha oposición, debido a que la nación ha estado sometida durante mucho tiempo a reglamentaciones que limitan todo tipo de libertades y derechos como la natalidad, el establecimiento del lugar donde deben vivir, donde deben estudiar, etc. Como se observa, estos son problemas de carácter estructural, por tanto, difíciles de superar (Cornejo, 2014, p. 3).

\section{ECONOMÍA Y POLÍTICA}

El desarrollo económico que ha experimentado China en los últimos quince años se ha debido a la implementación de una nueva estrategia diseñada para promover de forma sistemática las exportaciones, el acceso a los recursos primarios y el desarrollo de sus empresas. El rápido crecimiento de sus exportaciones los ha llevado a convertirse en el primer exportador del mundo, con reservas de moneda de más o menos de 3 billones de dólares; esto le ha permitido convertirse en el mayor comprador de bonos del tesoro de Estados Unidos, y ser el país con mayor inversión extranjera directa colocada en el mundo, unos 128.500 millones de dólares en el 2014, frente a los 92.000 millones que recibió Estados Unidos (UNCTAD, 2015; OECD, 2014).

En ese sentido, China ha venido oscilando entre el primer y segundo lugar como potencia económica mundial, con un PIB de más 
de 8 billones dólares. Por ejemplo, de acuerdo con la paridad del poder adquisitivo, China ocupó en 2015 el primer lugar con un valor de 19.510 billones de dólares (Factbook de la CIA), mientras que según informes oficiales chinos, en términos corrientes, es de 10.865 billones de dólares, ocupando el segundo lugar después de la de Estados Unidos (CIA, 2016; Banco Mundial, 2015). En todo caso, se debe advertir que esto no se refleja en el uso de su moneda fuera de sus fronteras ni en la distribución real de los ingresos entre la población. También ocupan el segundo puesto en consumo de petróleo a nivel mundial y, además, se han convertido en la tercera potencia exportadora del planeta en bienes y servicios.

Igualmente, es el primer país manufacturero del mundo ${ }^{3}$, gracias a que las empresas extranjeras han abierto sus fábricas en su suelo por los bajos costes de mano de obra y porque la legislación permitía gestionar y funcionar sin mayores requerimientos, lo que hacía que la operación fuera más fácil y rentable. No obstante, en los últimos años las buenas condiciones para las empresas y proporcionalmente injustas para la población trabajadora han cambiado; en consecuencia, los márgenes de ganancia de otras épocas han disminuido, al punto que muchas empresas han decidido buscar otros destinos, como Malasia y México, lo que ha llevado a que se dé una ralentización económica en China.
A pesar de este retroceso luchan por participar activamente en el orden internacional mediante acuerdos internacionales o reforzando los ya existentes. Por un lado, en el BRIC (Brasil, Rusia, India y China) se han mostrado desafiantes en sus cumbres anuales frente al dólar como moneda de reserva mundial y, por ende, reclaman un mayor rol en las instituciones financieras internacionales, aunque esto casi siempre ha quedado en papel mojado (Lacqueur, 2010; Stewart, 2010; Economy, 2010).

La crisis de la economía mundial los golpeó duramente en el quinquenio pasado, pero pudieron mantener una baja inflación $\mathrm{y}$ un crecimiento económico constante. Este segundo puesto le permite un activismo en política internacional a través de la cooperación internacional, llenando vacíos dejados por los poderes occidentales en África y en América Latina, lo que le ha permitido extender su influencia y ser aceptado y admirado, sobre todo en países abiertamente antiamericanistas. China también se ha convertido en el principal prestamista de muchos países latinoamericanos, y a través de los bancos han financiado a un grupo de países de la región que han dejado de recurrir al Banco Mundial, al Banco Interamericano de Desarrollo o, en general, a los bancos norteamericanos y europeos.

Es por eso que el expansionismo económico logrado por el Gobierno chino ha retado, aunque sin éxito, las reglas de juego

\footnotetext{
3 Las empresas manufactureras realizan actividades de transformación. Es decir, reciben materias primas que incorporan o complementan por medio de procesos y les agregan valor.
} 
planetarias creadas, promovidas y lideradas por Occidente, sobre todo en lo que concierne a la concepción de la soberanía como algo circunstancial, creencia que se ha visto reforzada desde la década de los noventa por las múltiples intervenciones internacionales justificadas desde el liberalismo, en concreto en nombre de la defensa de los derechos humanos y la ayuda humanitaria.

En ese sentido, las acciones emprendidas por China se han destinado a oponerse al intervencionismo en Sudán, Somalia, Haití o Bosnia (Lyons y Mastanduno, 1995; Stewart, 2010); también a la guerra global contra el terrorismo a la manera como la emprende Estados Unidos contra dictadores sanguinarios, o se opone a que se viole la soberanía de los Estados sospechosos de producir o comercializar armas de destrucción masiva. Además, junto con otras potencias emergentes, como Brasil, India, Sudáfrica y ahora Turquía, han votado en contra de las medidas del Consejo de Seguridad de la ONU referentes a las violaciones de los derechos humanos en Birmania, Sri Lanka, Sudán o Zimbabwe (Stewart, 2010).

Es por eso que ya no confiere a Estados Unidos la potestad para decidir los límites de una soberanía responsable; reforzando el acercamiento entre los regímenes autoritarios de la región, la Organización para la Cooperación de Shanghái, liderada por Rusia y China, ha endurecido su posición hacia Estados Unidos, mediante la contención de su presencia en Asia Central (Brown y Ainley, 2009).

Sin embargo, es muy diciente que el resultado del asombroso crecimiento de su economía haya impulsado, aunque prudentemente, al Gobierno chino a revivir su po- lítica de dominio estratégico de las islas y los mares circundantes, no solo con el propósito de consolidar la clásica "política del poder" marítimo y mundial, sino también para crear una zona de paso segura para todos sus buques mercantes (Economy, 2010). Prudentemente, porque no es probable que ni en el corto ni en el mediano plazo esta "expansión estratégica” de China logre los resultados deseados, pues esta política tropezará con la resistencia de Estados Unidos, Japón, Corea del Sur, Vietnam, Indonesia, entre otros países que circundan el Mar de China y perciben el peligro para su seguridad nacional por la eventual expansión china.

Prudentemente, además, porque China es consciente de que no puede amenazar seriamente la supremacía militar de Estados Unidos y ni siquiera la de vecinos como Japón. En realidad, la política internacional china ha sido pragmática frente al uso del poder que le ha dado Estados Unidos al manejo de los asuntos globales: debido a su interdependencia económica y a la necesidad de preservar el mercado estadounidense, China ha preferido mantener una actitud "neutral" en el frente occidental liderado por Estados Unidos en la reorganización político-militar del mundo y especialmente en la lucha contra el terrorismo (Domenach, 2009; Cypel, 2009, p. 35).

Más aún, junto a Moscú, preparan para presentar a la ONU un proyecto de resolución denominada Medidas de Transparencia y Confianza en las Actividades Espaciales, que contempla prohibir el despliegue de armas en el espacio exterior. Esto demuestra la intención de no ir a una confrontación militar directa, aunque la tensión aumenta considerablemente 
si se tiene en cuenta la alianza militar que Estados Unidos tienen con Japón, un rival histórico de China. En el siglo Xxi se muestra más dispuesta a desafiar moderadamente el orden mundial establecido y liderado por Estados Unidos; una muestra de ello es que no apoyó la invasión a Irak en 2003 y continúa con éxito con su régimen autocrático (Stewart, 2010, Economy, 2010).

Por su parte, Estados Unidos prefiere concertar mecanismos de "acercamiento" con China -y no emprender una política de “provocación”-, porque esa actitud es más conveniente para conseguir sus objetivos nacionales - un impulso egoísta donde los Estados piensan primero en sus ganancias-. Por otro lado, es consciente de que China no tiene ni la capacidad ni la intención para rivalizar militarmente con la potencia hegemónica, y se acomodan a las condiciones del sistema mientras logran el equilibrio; y en el de la potencia hegemónica justamente por su declive (Estados Unidos se encuentra en una fase de declive relativo), y por tanto, a la incapacidad para hacer frente a ese tipo de situaciones unilateralmente (Waltz, 2000; Mearsheimer, 1995; Grieco; 1988; Layne, 2012).

Vale la pena detenerse a analizar el vínculo entre la reacción de Estados Unidos y la presencia de China en regiones como América Latina, ya que la expansión de este país impacta significativamente en la política y la economía estadounidense en su zona de influencia directa y sobre sus socios de la región. Lo que llama la atención, sin embargo, es que las relaciones chino-norteamericanas en torno a las zonas de influencia directa de Estados Unidos revelan que ambos paísessacan ventaja del rol que cada uno desempeña. En otras palabras, Estados Unidos no tiene muchos motivos o fundamentos para oponerse radicalmente a la expansión de la influencia china en el corto plazo; más aún, tanto China como Estados Unidos tienen razones de sobra para esperar que ciertas zonas, especialmente América Latina, sean más prósperas, para que les pueda asegurar un mercado más amplio.

No obstante, la expansión de China puede ser explicada desde la geoestrategia, esto debido a la necesidad de ese país de contener la estrategia intervencionista de Estados Unidos en una región que China considera como su propia esfera de influencia, esto es, Asia-Pacífico, lo que a su vez es consecuencia de la estrategia de política exterior del presidente Obama de "reequilibrarse" hacia Asia. China ve ese movimiento como una amenaza a su seguridad.

En ese caso, si China percibe que Estados Unidos es o tiene una presencia mayor en su propio patio trasero de Asia Oriental, aumentaría o utilizaría los recursos con los que ya cuenta en regiones de influencia estadounidense para desafiar la hegemonía norteamericana; en ese sentido, es probable que en el largo plazo los Estados con los que China tiene una relación muy estrecha se conviertan en "clientes" de la potencia asiática, disminuyendo así la presencia y dependencia que estos tienen con Estados Unidos.

Esas relaciones no están basadas en una variable ideológica, sino en una política pragmática cuyo fundamento de política exterior se circunscribe a la estrategia china de emprender relaciones "de pueblo a pueblo"; este comportamiento está basado en su doctrina 
de política exterior de la no intervención y la promoción de la paz mundial. Por tal razón, China prefiere invertir o ampliar sus relaciones en aquellos países que tienen cierta estabilidad económica o que pueden ofrecer su respaldo diplomático para implementar su agenda internacional en el mundo. Por otro lado, hay países que encuentran una alternativa viable en la profundización de sus relaciones con China para desprenderse de Estados Unidos. La principal variable ideológica que tiene en cuenta China para emprender relaciones con otros países es que estos no reconozcan a Taiwán.

En la actual configuración de la política internacional, estas relaciones se constituyen en un mecanismo estratégico para China y sus socios que buscan un cambio en la distribución de poder, con preferencia un sistema multipolar con un orden basado en la pluralidad y el equilibrio en la implantación de las reglas y los principios de relacionamiento entre los diversos Estados.

La sociedad estratégica que ha conseguido China, sobre todo con aquellos países que tienen una política antinorteamericana, no implica la implementación de alianzas militares o el apoyo directo en caso de una guerra. Por ahora, solo se puede pronosticar que esa alianza tendría efectos más diplomáticos, por ejemplo, en apoyo político en el marco de las organizaciones internacionales, posibles sanciones por parte de Estados Unidos o la ONU, y en general para respaldar o asegurar su neutralidad en caso de eventuales intervenciones internacionales sobre esos Estados.

Finalmente, en las actuales circunstancias, China utiliza el poder blando junto al duro para intentar transformar la actual distribución de poder, con la aspiración, a largo plazo, de desafiar la hegemonía estadounidense a través de la movilización de elementos materiales e ideacionales.

\section{CONCLUSIÓN}

Actualmente China es la segunda potencia económica del mundo y será rápidamente, según los cálculos de expertos, la primera en volumen de producto interno bruto. Sin embargo, es contradictorio que exista un deficiente Estado de bienestar para la mayoría de sus ciudadanos, situación que explica el constante flujo migratorio extraterritorial.

Por tales razones, tiene el reto de garantizar el acceso a fuentes primarias, desarrollar actividades tendientes a la generación de capital, asegurar la capacidad alimentaria de sus ciudadanos en la medida en que se urbaniza y aumenta el consumo de más alimentos, construir y amparar mercados para sus productos mientras aumenta su capacidad productiva y de valor agregado. A nivel internacional, asegurar acceso a los flujos de tecnología e información global y mantener una presencia en instituciones claves para las transacciones económicas globales.

En todo caso, la política que ha implementado internacionalmente es más pragmática que desafiante, porque busca consolidar sus objetivos nacionales sin retar directamente la hegemonía estadounidense, asegurando, por ahora, el acceso a materias primas y un mercado para sus productos.

Finalmente, China requiere solucionar todos sus problemas internos para lograr 
una nueva distribución de poder, al menos un sistema multipolar clásico, para evitar la consolidación de una coalición internacional que se oponga a su resurgimiento.

\section{REFERENCIAS}

Anguiano, E. (1999). Medio siglo de vida de la República Popular China: nueva historia milenaria. Conferencia, septiembre de 1999.

Banco Mundial (2015). Datos. Washington: Grupo del Banco Mundial.

Brown, C. y Ainlay, K. (2009). Understanding International Relations. New York: Palgrave-Macmillan.

CIA (2016). China. Recuperado de https://www.cia. gov/library/publications/the-world-factbook/ geos/ch.html

Cypel, S. (2009). Obama, del hard power al soft power. En Bertrand, B. y Dominique, V. (dirs.). El Estado del Mundo. Anuario económico y geopolítico mundial, 2010 (pp. 34-38). Madrid: Akal.

Cornejo, R. (2014). La relación de México con China: de la política del desconcierto al acercamiento diplomático. Ciudad de México: Colegio de México.

Domenach, J. (2009). Una China más poderosa y mejor integrada. En Bertrand, B. y Sandrine, T. (dirs.). El Estado del Mundo. Anuario económico y geopolítico mundial, 2010 (pp. 39-43). Madrid: Akal.

Ellis, E. (2013). The Strategic Dimension of Chinese Engagement with Latin America. Washington: Center for Hemispheric Defense Studies.

Elis, E. (2009). China in Latin America: The Whats and Wherefores. Boulder: Lynne Rienner Publishers.

García, P. (2001). El regreso del dragón, geopolítica de Asia y el Pacífico. Bogotá: Universidad Externado de Colombia.
Grieco, J. (1988). Anarchy and the Limits of Cooperation: A Realist Critique of the Newest Liberal Institutionalism. International Organization, 42 (3). Human Rights Watch (2014). World Report 2013. Recuperado de https://www.hrw.org/worldreport/2013

Lacqueur, W. (2010). Moscow's Modernization Dilemma: Is Russia Charting a New Foreign Policy? Foreign Affairs, 89 (6), 153-162.

Jae-kyung Park (2013). China-U.S. Relations in East Asia. Strategic rivalry and korea's choice. Washington: Center for Strategic and International Studies. Recuperado de http://csis.org/files/publication/130404_Park_ChinaUSRelations_Web.pdf

Layne, C. (2012). The (Almost) Triumph of Offshore Balancing. The National Interest. Recuperado de http://nationalinterest.org/commentary/almosttriumph-offshore-balancing-6405

Lyon, G. y Mastanduno, M. (1995). International Intervention, State Sovereignty, and the Future of International. Baltimore: The John Hopkins Universtiy Press.

Mearsheimer, J. (1995a). The False Promise of International Institutions. International Security, 19 (3).

Mearsheimer, J. (1995b). Society. En Lyons, G y Mastanduno, M. (eds.). Beyond Westphalia. State Sovereignty and International Intervention. Baltimore: The John Hopkins Universtiy Press.

OECD (2014). International investment struggles. Recuperado de http://www.oecd.org/daf/inv/investment-policy/FDI-in-Figures-Feb-2014

Stewart, P. (2010). Irresponsible Stakeholders? The Difficulty of Integrating Rising Powers. Foreign Affairs, 89 (6), 44-55.

Pérez, J. G. (2012). El mapa de los conflictos en Asia. Investigaciones Geográficas, 39-53. 
Quansheng, Z. (2001). Modernization, Nationalism, and Regionalism in China. Washington: American University.

Sun, Y. (2013). March West: China's Response to the U.s. Recuperado de http://www.brookings.edu/blogs/ up- front/posts/2013/01/31-china-us-sun

unCTAD (2015). Country Fact Sheets 2015. Recuperado de http://unctad.org/en/Pages/DiaE/World\%20 Investment\%20Report/Country-Fact-Sheets. aspx

Velosa, E. (2010). ¿¿Asia Pacífico es más que economía?: la necesidad de una política exterior integral.
En Más allá de la seguridad democrática. Agenda hacia nuevos horizontes. Bogota: Pontificia Universidad Javeriana.

Waltz, K. (2000). Structural Realism after the Cold War. International Security, 25 (1).

World Bank. (2015). Data. GDP growth (annual \%). Recuperado de http://data.worldbank.org/indicator/NY.GDP.MKTP.KD.ZG

Wu Xinbo (2011). China and the United States Core Interests. Common Interests, and Partnership. United States Institute of Peace. Recuperado de http://www.usip.org/sites/default/files/sR277.pdf 